\title{
Early dissociation between neural signatures of endogenous spatial attention and perceptual awareness during visual masking
}

\author{
Valentin Wyart ${ }^{1,2,3}$, Stanislas Dehaene ${ }^{4,5,6}$ and Catherine Tallon-Baudry ${ }^{1,2,3,7}$ \\ ' Centre de Recherche de I'Institut Cerveau-Moelle (CRICM), Unité Mixte de Recherche CNRS/INSERM, Université Pierre et Marie Curie, Paris, France \\ ${ }^{2}$ Centre National de la Recherche Scientifique (CNRS), UMR 7225, CRICM, Université Pierre et Marie Curie, Paris, France \\ ${ }^{3}$ Institut National de la Santé et de la Recherche Médicale (INSERM), UMR S-975, CRICM, Université Pierre et Marie Curie, Paris, France \\ ${ }^{4}$ Unité de Neuroimagerie Cognitive CEA/INSERM, Centre NeuroSpin, Université Paris Sud, Gif-sur-Yvette, France \\ ${ }^{5}$ Commissariat à l'Energie Atomique (CEA), Direction des Sciences du Vivant, Centre NeuroSpin, Gif-sur-Yvette, France \\ ${ }^{6}$ Collège de France, Paris, France \\ ${ }^{7}$ Centre de Neuroimagerie de Recherche (CENIR), Hôpital de la Pitié-Salpêtrière, Paris, France
}

\section{Edited by:}

Hans-Jochen Heinze, University of Magdeburg, Germany

Reviewed by:

Douglas O. Cheyne, Hospital for Sick Children, Canada

Carsten N. Boehler, Leibniz Institute for Neurobiology, Germany

\section{*Correspondence:}

Valentin Wyart, Department of

Experimental Psychology, University

of Oxford, South Parks Road,

Oxford, OX1 3UD, UK.

e-mail: valentin.wyart@gmail.com
The relationship between spatial attention and conscious access has often been pictured as a single causal link: spatial attention would provide conscious access to weak stimuli by increasing their effective contrast during early visual processing. To test this hypothesis, we assessed whether the early attentional amplification of visual responses, around $100 \mathrm{~ms}$ following stimulus onset, had a decisive impact on conscious detection. We recorded magnetoencephalographic (MEG) signals while participants focused their attention toward or away from masked stimuli which were physically identical but consciously detected half of the time. Spatial attention increased the amplitude of early occipital responses identically for both detected and missed stimuli around $100 \mathrm{~ms}$, and therefore, did not control conscious access. Accordingly, spatial attention did not increase the proportion of detected stimuli. The earliest neuromagnetic correlate of conscious detection, around $120 \mathrm{~ms}$ over the contralateral temporal cortex, was independent from the locus of attention. This early activation combined objective information about stimulus presence and subjective information about stimulus visibility, and was followed by a late correlate of conscious reportability, from $220 \mathrm{~ms}$ over temporal and frontal cortex, which correlated exclusively with stimulus visibility. This widespread activation coincided in time with the reorienting of attention triggered by masks presented at the uncued location. This reorienting was stronger and occurred earlier when the masked stimulus was detected, suggesting that the conscious detection of a masked stimulus at an unexpected location captures spatial attention. Altogether, these results support a double dissociation between the neural signatures of endogenous spatial attention and perceptual awareness.

Keywords: magnetoencephalography, event-related responses, neural oscillations, visual perception, attention, consciousness, decision making

\section{INTRODUCTION}

Attention is often considered as a gateway for awareness (Baars, 1988; Dennett, 1991; Dehaene et al., 2006): because sensory responses to attended stimuli are enhanced, those attended stimuli are more likely to reach the threshold for awareness. This view is supported by numerous psychophysicial studies showing that attention enhances contrast sensitivity and appearance (Bashinski and Bacharach, 1980; Carrasco et al., 2004; Liu et al., 2009; Störmer et al., 2009; for a review, see Carrasco, 2011). From a mechanistic point of view, top-down attention does enhance neural sensitivity in monkey visual areas (McAdams and Maunsell, 1999; Reynolds et al., 2000; Martinez-Trujillo and Treue, 2002). In humans, top-down attention acts by suppressing alpha band oscillations in visual areas contralateral to the attended location (e.g., Worden et al., 2000; Thut et al., 2006; Siegel et al., 2008), and by enhancing sensory responses to attended stimuli in extra striate visual areas between 80 and $180 \mathrm{~ms}$ (for reviews, see Hillyard et al., 1998; Luck et al., 2000). The two measures are related and correlate with behavior (Gould et al., 2011). It could, therefore, be predicted that endogenous attention facilitates conscious perception by amplifying sensory responses.

However, this simple relationship between spatial attention and perceptual awareness is currently being reconsidered from a theoretical point of view (Lamme, 2003, 2004; Dehaene et al., 2006; Koch and Tsuchiya, 2007), and several lines of experimental evidence support the idea of a dissociation between the two processes. The first hint for a behavioral dissociation between endogenous spatial attention and perceptual awareness 
was obtained in a "blindsight" patient (Weiskrantz, 1986), who shows a significant speeding of reaction times to cued, yet unperceived stimuli presented in his blind visual field (Kentridge et al., 2004). A number of behavioral experiments now report attentional modulations in the absence of consciousness in normal subjects (Sumner et al., 2006; Kentridge et al., 2008; Kiefer and Martens, 2010; Faivre and Kouider, 2011; Martens et al., 2011), or even a double dissociation between attention and consciousness (van Boxtel et al., 2010). At the neural level, there is growing evidence that attention can modulate electrophysiological responses to stimuli that do not reach consciousness (Naccache et al., 2002; Woodman and Luck, 2003; Kiefer and Brendel, 2006; Koivisto et al., 2006; Koivisto and Revonsuo, 2007, 2008). Neural correlates of consciousness can also appear quite independent from attention (Koivisto et al., 2006; Boehler et al., 2008). A double dissociation of the spectral fingerprints of spatial attention and perceptual awareness has even been obtained in the visual cortex of healthy participants (Wyart and Tallon-Baudry, 2008), as well as in a blindsight patient (Schurger et al., 2008). However, this double dissociation was observed on non-phase-locked visual responses occurring more than $200 \mathrm{~ms}$ following stimulus onset, using low-contrast gratings which evoked no measurable phaselocked responses at earlier latencies. Whether or not the early attentional amplification of visual responses fosters awareness, therefore, remains an open issue.

To address this issue, we impaired the visibility of the gratings not solely by lowering their contrast, but by replacing them with a visual mask $50 \mathrm{~ms}$ after onset. This backward masking procedure has two clear advantages: (1) undetected gratings can still be of high contrast, and therefore, can reliably trigger early visual responses before $200 \mathrm{~ms}$, and (2) while lowering the contrast of stimuli imposes a signal-strength constraint on detection, masking the same stimuli imposes a temporal constraint on detection. Indeed, most theories of visual masking propose an interruption of target processing by mask processing (Breitmeyer and Öğmen, 2006): it is thus likely the earliest visual responses to the masked target that have a decisive impact on its detection. We, therefore, focused our analyses on early visual responses phase-locked to the onset of the masked targets, to test whether or not the early attentional amplification of visual processing bears a tight relationship with conscious access.

Therefore, we recorded magnetoencephalographic (MEG) signals while human participants focused their attention toward or away from a single masked visual grating which was consciously detected only half of the time (Figure 1). At the beginning of each trial, participants were cued by a predictive central arrow to covertly attend either to their left or right lower visual field (Posner et al., 1980). After $600 \mathrm{~ms}$, a target grating at detection threshold was briefly presented for $17 \mathrm{~ms}$ either at the cued or uncued location, or occasionally was not presented at all (see Materials and Methods), and was followed after $50 \mathrm{~ms}$ by a high-contrast, unilateral metacontrast mask, surrounding without overlapping the location of the target (Breitmeyer and Öğmen, 2006; Ishikawa et al., 2006). After mask offset, participants performed two delayed tasks: first, they selected the perceived orientation of the target grating among two choices (discrimination task), and then they reported whether they had

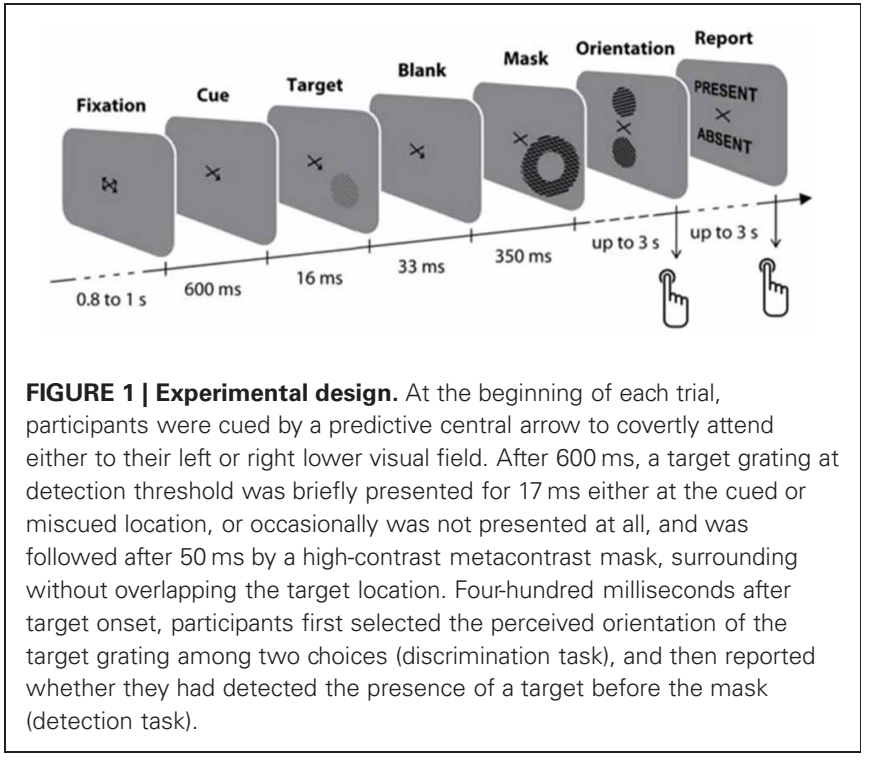

detected the presence of a target before the mask (detection task). We assessed at which times target-driven neuromagnetic responses were modulated by spatial attention, and whether the earliest attentional amplification of target processing critically determined its conscious detection.

\section{MATERIALS AND METHODS PARTICIPANTS}

Sixteen participants with normal or corrected-to-normal vision took part in the study. All participants provided informed written consent and were paid $60 €$ for their participation. All procedures were approved by the local ethics committee (Comité Consultatif de Protection des Personnes dans la Recherche Biomédicale). Four participants were excluded from the analysis because of irrepressible eye movements toward the mask $\left(>1.0^{\circ}\right.$ of visual angle from fixation). The remaining twelve participants were all righthanded and naive to the purpose of the experiment (eight males, age range: $19-28$ years).

\section{STIMULI AND BEHAVIORAL TASK}

Visual targets were circular gratings (spatial frequency: 5.0 cycles per degree of visual angle, diameter: $2.0^{\circ}$ of visual angle, orientation: chosen among 20 equally spaced between $0^{\circ}$ and $180^{\circ}$, cardinal orientations being excluded). Targets were positioned at $2.5^{\circ}$ of visual angle from fixation with a declination of $30^{\circ}$ in the left or right lower visual quadrants to maximize early neuromagnetic visual responses (Portin et al., 1999). The metacontrast masks were two superimposed annulus gratings surrounding the targets without overlapping them (spatial frequency: 5.0 cycles per degree of visual angle, inner diameter: $2.0^{\circ}$ of visual angle, outer diameter: $4.0^{\circ}$ of visual angle, orientations: the orientation of the target, and another orientation distant from $60^{\circ}$ from the orientation of the target). The contrast of the targets was manipulated on a fine-grained basis using a simple software attenuator (Tyler, 1997). The left and right spatial cues only differed by three arrow tips from the fixation cross (Figure 1) and were subtending $0.9^{\circ} \times 0.6^{\circ}$ of 
visual angle around fixation. The stimuli, fixation cross and spatial cues were created offline using MATLAB (The MathWorks, Natick, MA) and stimulus presentation was controlled using the Psychtoolbox-3 package for MATLAB (Brainard, 1997; Pelli, 1997). All stimuli were presented via a mirror system on a gray background (luminance: $30.0 \mathrm{~cd} / \mathrm{m}^{2}$ ) at the center of a back projection screen positioned at $85 \mathrm{~cm}$ from the participant's eyes, using a gamma-corrected video projector (resolution: $1024 \times 768$ pixels, refresh rate: $60 \mathrm{~Hz}$ ) located outside the shielded recording room. The luminosity of the recording room was controlled as well as the luminance of the gray background of the projection screen.

Each participant underwent a calibration session prior to the experiment in order to estimate the threshold contrast for which only half of the masked stimuli (constant SOA: $50 \mathrm{~ms}$ ) were reported as present. The calibration session was followed by eight recording sessions. Each of the eight recording sessions consisted of 92 trials, including 80 "target-mask" trials in which target at threshold contrast were presented either in the left or right lower visual field and followed by a single annulus metacontrast mask surrounding the target location, and 12 "mask-only" trials in which only the metacontrast mask was presented. Fiftytwo of the 80 targets were presented at the cued location, while 28 were presented at the uncued location (i.e., miscued). Trials within a recording session were presented in a different pseudorandomized order for each participant. Participants were not given any explicit feedback regarding either the proportion of target-absent trials or the presence of a target at the end of each trial, since such information could have introduced undesirable prior expectations about the presence of a stimulus in subsequent trials. After $400 \mathrm{~ms}$, participants were first asked to discriminate as quickly and accurately as possible the orientation of the target among two choices distant by $60^{\circ}$. Participants pressed the upper response button with their index finger to choose the upper orientation or the lower response button with their middle finger to choose the lower orientation. When they did not see the target, participants were asked to guess. After a manual response, or after $3 \mathrm{~s}$ without response $(<1 \%$ of all trials), participants were asked to report whether or not they had detected the presence of a target before the mask during the trial. The words "present" and "absent" were presented vertically, their respective positions being randomized across trials. Participants were asked to report "present" when they believed that a target had been presented, whether they could discriminate its orientation or not, and choose "absent" when they believed that no target had been presented before the mask. Participants pressed the upper response button with their index finger to choose the upper report or the lower response button with their middle finger to choose the lower report. After a variable delay of 2-3s following the participant's response, the next trial began.

We have used the variable response mapping scheme described above in order to avoid response preparation brain signals in our post-stimulus analysis period (i.e., before the onset of the discrimination response prompt). However, the use of response prompts resulted in delayed response times, which in turn could have strongly diminished their sensitivity to cognitive factors such as spatial attention when compared to conventional cueing paradigms (e.g., Posner et al., 1980).

The first judgment should be seen as a forced-choice discrimination task. This forced-choice strategy is often used to look for subliminal effects, i.e., higher-than-chance performance despite a subjective lack of perception. Subjects underwent the forcedchoice judgment before the subjective present/absent report because, when they exist, subliminal effects are usually short-lived and very sensitive to distraction. So if we had asked the subjective report first, the lack of a subliminal effect on discrimination accuracy could have been overshadowed by its greater temporal distance from target presentation.

\section{MEG RECORDINGS}

Continuous magneto-encephalographic signals were collected using a whole-head MEG system with 151 axial gradiometers (CTF Systems, Port Coquitlam, BC: Canada) at a sampling rate of $1250 \mathrm{~Hz}$ and low-pass filtered online at $300 \mathrm{~Hz}$. Head localization was tracked during the experiment with respect to the MEG sensor array using marker coils that were placed at the cardinal points of the head (nasion, left, and right ear). Calibrated vertical and horizontal electrooculogram (EOG) signals were simultaneously collected.

\section{DATA ANALYSIS}

Data analysis was performed using in-house software, the Fieldtrip package, and custom programs developed for MATLAB (The MathWorks, Natick, MA). All time samples were corrected with respect to the refresh delay of the projector $(+25 \mathrm{~ms}$, measured online with a photodiode). For the sensor-level analysis, orthogonal planar gradients of the MEG field distribution were computed using a nearest-neighbor method (Bastiaansen and Knosche, 2000). The horizontal and vertical components of the estimated planar gradients approximate the signal measured by MEG systems with planar gradiometers. The planar gradients are typically largest in magnitude directly above a given source, which is particularly advantageous when interpreting topographical distributions of MEG activity at the sensor level (see, e.g., Bauer et al., 2006; Osipova et al., 2006; Jokisch and Jensen, 2007). Gradiometers signals were averaged across trials, baseline corrected and planar-transformed, and finally combined by taking their vector norm to analyze event-related fields. Switching from axial signals to planar gradients: (1) improved the signal-to-noise ratio of visual responses across conditions when averaging across subjects, and (2) revealed qualitative differences in the dynamics of visual responses between nearby MEG sensors that were completely invisible when using axial signals. Importantly, this planar transformation forbids the identification of successive dipole reversals, which could have been used as an alternative strategy to identify visual components and their underlying neural sources using axial signals (e.g., Noesselt et al., 2002). However, because targets and masks were displayed in rapid succession (spaced by $50 \mathrm{~ms}$ ), the classical peaks of the visual evoked response to a single stimulus could not be identified. This precluded the use of the dipole-reversal strategy to analyse the data, and argued in favor of the planar transformation. 


\section{Artifact rejection}

Trials contaminated with eyes movements (rejection threshold: $1.0^{\circ}$ of visual angle from fixation), eye blinks or muscular artifacts were rejected offline upon visual inspection of their unfiltered EOG and MEG traces. Furthermore, one malfunctioning MEG sensor $\left(\mathrm{RT}_{32}\right.$ overlying right temporal cortex) was discarded prior to all analyses, and a high-pass filter at $0.1 \mathrm{~Hz}$ was applied. Besides, phase-locked analyses of visual analyses were performed following a low-pass filter at $40 \mathrm{~Hz}$.

\section{Time-frequency analysis}

A time-frequency wavelet transform was applied to each trial at each interpolated planar MEG sensor using a family of complex Morlet wavelets $(m=10)$, resulting in an estimate of oscillatory power at each time sample and at each frequency between 5 and $30 \mathrm{~Hz}$. The parameter $m$ refers to the ratio between frequency and the half-width in the spectral domain $m=\mathrm{f} / \sigma_{f}$ (e.g., Tallon-Baudry et al., 1997). The width of the wavelet in the time domain is with $2 \sigma_{t}=1 /\left(\pi \sigma_{f}\right)$. At $10 \mathrm{~Hz}, m=10$ corresponds to a $2 \sigma$-duration of $318 \mathrm{~ms}$. In contrast to eventrelated fields, gradiometer signals were planar- and wavelettransformed at the single-trial level, and finally averaged across trials. The time-frequency data during the last $200 \mathrm{~ms}$ of the fixation period preceding each trial (200-0 ms before cue onset, i.e., $800-600 \mathrm{~ms}$ before stimulus onset) was used as baseline. An index of signal power, defined at each time sample and each frequency as the increase of spectral power relative to fixation baseline in logarithmic decibel units, was considered as the measure of interest for all time-frequency analyses. Indeed, the log-transformed data were distributed normally, which allowed us to use standard parametric tests (e.g., paired $t$-test, repeatedmeasures ANOVA) to assess the statistical significance of observed effects (Kiebel et al., 2005). Importantly, we did not subtract the averaged evoked field from each single trial before computing time-frequency power.

\section{Peak latency analysis}

Peak latencies of phase-locked visual responses were estimated semi-automatically by locating negative zero-crossings of the first-order derivative of planar MEG gradiometer traces (lowpass filtered at $40 \mathrm{~Hz}$ ) between 100 and $200 \mathrm{~ms}$ following stimulus onset (i.e., from 50 to $150 \mathrm{~ms}$ following mask onset). Peak latencies were visually inspected for outliers for each subject and condition.

\section{Baselining procedures}

When studying post-stimulus effects (i.e., following the onset of the target at $0 \mathrm{~ms})$, we have used a pre-cue baseline $(-800$ to $-600 \mathrm{~ms}$ relative to target onset, i.e., -200 to $0 \mathrm{~ms}$ relative to cue onset) where there was no difference between conditions in the pre-target period ( -200 to $0 \mathrm{~ms}$ relative to target onset). Where there was a significant difference between conditions of interest in the pre-target period, we have adopted a pre-target baseline ( -200 to 0 relative to target onset) as a conservative means to test for an additional post-stimulus difference between conditions that would not simply be the continuation of an existing pre-stimulus difference. Importantly, peak latency analyses are free from baselining because they are based on the first-order derivative of MEG signals.

\section{Statistical procedures}

We performed a non-parametric permutation test to assess whether the early lateralization effect (at 90-110 ms following target onset) observed in target-mask trials but not in mask-only trials could be merely due to the higher number of target-mask trials than mask-only trials. At the single-subject level, we randomly split all trials in two sets with unbalanced numbers of trials matching the numbers of trials found in the target-mask and mask-only conditions, respectively. Importantly, the proportions of target-mask and mask-only trials were made equal in the two trial sets. At the group level, we then tested the interaction between the lateralization effects found in the two random trial sets using a repeated-measure ANOVA, and stored the corresponding $p$-value. We repeated this procedure 10,000 times, and assessed whether the $p$-value of the original interaction was lower than the $5 \%$ quantile of the $p$-value distribution obtained through permutations. The corrected $p$-value for the interaction corresponds to the proportion of permuted $p$-values smaller than the original $p$-value.

\section{Topographical visualization procedures}

We have used a straightforward procedure in order to plot the data from both left and right targets on a single topographical scalp map. To do so, we: (1) flipped the MEG sensor labels between the left and right hemispheres for trials corresponding to right-lateralized stimulation, and (2) averaged left-lateralized and flipped right-lateralized trials for each subject and condition. As a result, all the topographical maps presented in the figures show the sensors contralateral to the stimulation side on the right hemisphere, and the sensors ipsilateral to the stimulation side on the left hemisphere.

\section{RESULTS \\ BEHAVIOR}

Subjective reports were not made randomly: participants reported the presence of a target much more often when the target was actually present (hit rate: $51.0 \pm 1.7 \%$, mean \pm standard error) than absent (false-alarm rate: $10.9 \pm 2.7 \%$ ), corresponding to a positive detection sensitivity $d$ ' of $1.40 \pm 0.16$ ( $t$-test against zero, $p<0.001)$ along with a conservative decision bias $\beta$ of $+1.05 \pm 0.18$ in logarithmic units ( $t$-test against zero, $p<$ 0.001). Although participants could detect the presence of the masked target significantly better than chance, they could not discriminate the orientation of the masked target better than chance (accuracy: $50.2 \pm 1.0 \%, t$-test against chance, $p>0.5$ ), even when considering detected targets only $(51.0 \pm 1.3 \%, t$-test against chance, $p>0.2$ ). The detection and orientation discrimination $d$ ' were not correlated across participants (linear correlation test, $r^{2}<0.05, p>0.2$ ), in contrast to what we previously observed for non-masked stimuli (Wyart and Tallon-Baudry, 2008, 2009).

Spatial attention had no significant effect on hit rate (cued: $49.0 \pm 2.5 \%$, miscued: $49.5 \pm 2.7 \%$, paired $t$-test, $p>0.5$ ), falsealarm rate (cued: $10.0 \pm 2.9 \%$; miscued: $12.5 \pm 3.0 \%$, paired 
$t$-test, $p=0.12$ ), nor on detection sensitivity (cued: $1.40 \pm 0.21$, miscued: $1.21 \pm 0.17, p=0.15)$. Spatial attention had also no measurable effect on detection reaction times (cued: $763 \pm 39 \mathrm{~ms}$, miscued: $771 \pm 32 \mathrm{~ms}, p>0.5$ ), nor on discrimination reaction times (cued: $798 \pm 52 \mathrm{~ms}$, miscued: $776 \pm 49 \mathrm{~ms}, p>0.2$ ). No significant difference was found when further considering detected and missed targets separately.

Together, these behavioral results indicate that participants were able to detect the presence of the metacontrast-masked target better than chance, but could not discriminate its identity better than change (see also Vorberg et al., 2003). With those specific experimental conditions, we found that endogenous spatial attention did not significantly improve the detectability of the masked target, as previously reported for unmasked targets in the absence of spatial uncertainty (Gould et al., 2007).

\section{SELECTION OF RETINOTOPICALLY DEFINED REGIONS-OF-INTEREST}

To define regions-of-interest (ROIs) a priori (i.e., independently from spatial attention and perceptual awareness), we looked for lateralization effects on the evoked neuromagnetic responses relative to visual stimulation (contralateral minus ipsilateral to visual stimulation), and identified visually three bilateral ROIs (occipital, parietal and temporal) of 8 MEG sensors each which differed in terms of the time courses of their lateralization effects to visual stimulation (Figure 2, left). This selection criterion was used to identify bilateral MEG sensors that showed visual responses contralateral to the stimulation side, i.e., where the visual processing of the target is likely to take place.

At the occipital ROI (Figure 2A), an early transient lateralization to visual stimulation was observed at 90-200 ms following target onset (contralateral versus ipsilateral sensors, paired $t$-test, $p<0.001$ ). At the parietal ROI (Figure 2B), the early transient lateralization was observed at 100-170 ms following target onset (paired $t$-test, $p=0.001$ ), but was followed by a second sustained lateralization to visual stimulation at $210-480 \mathrm{~ms}$ (paired $t$-test, $p=0.001$ ). At the temporal ROI (Figure 2C), a weaker transient lateralization to visual stimulation was observed at $110-150 \mathrm{~ms}$ following target onset (paired $t$-test, $p<0.01$ ).

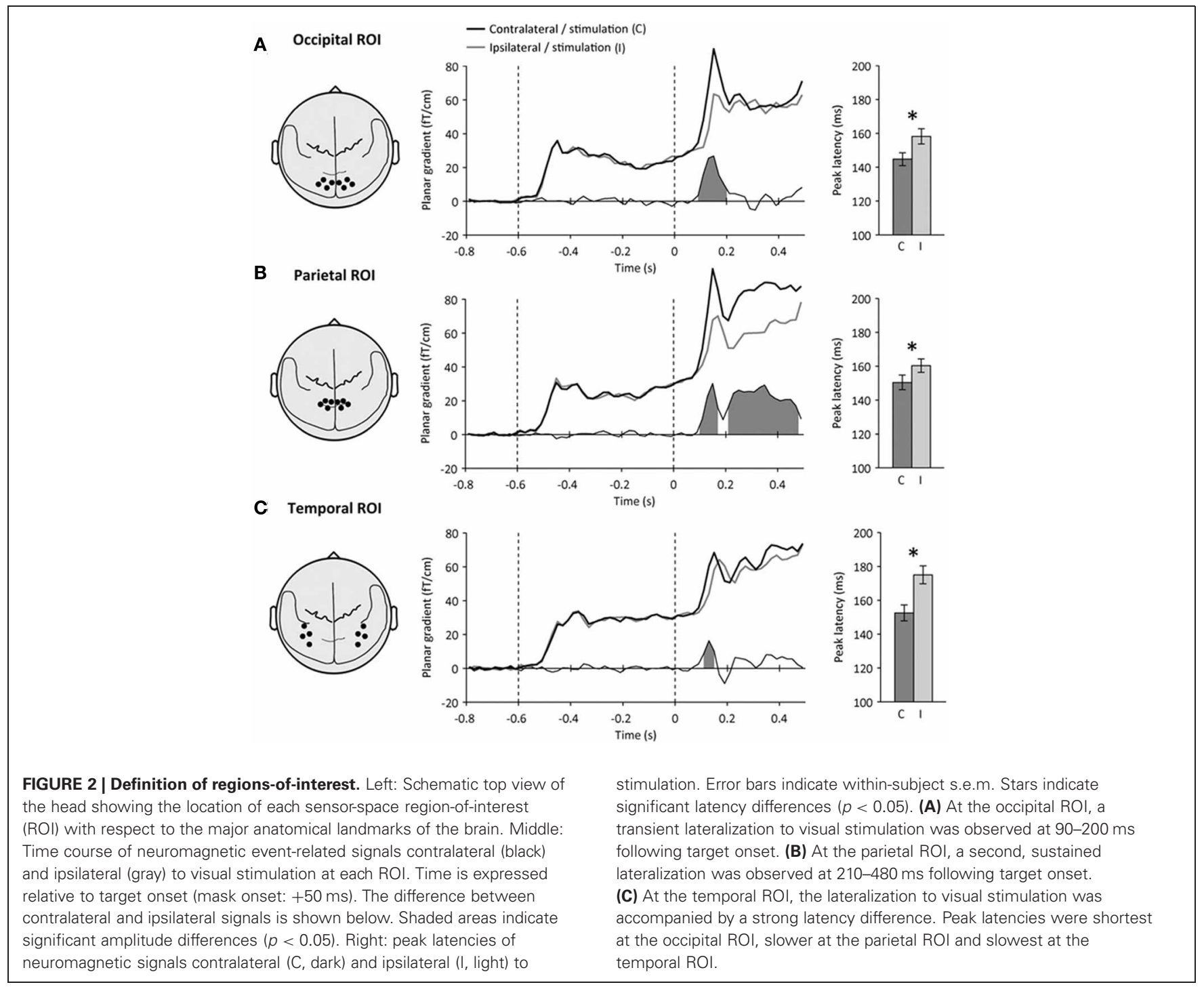


Occipital, parietal and temporal ROIs also differed in terms of the peak latencies of neuromagnetic responses to visual stimulation (Figure 2, right). Peak latencies differed significantly both between ROIs (repeated-measures ANOVA, main effect: ROI, $p=0.01$ ), and between contralateral and ipsilateral sensors relative to visual stimulation (main effect: hemisphere, $p<0.005$ ). Peak latencies were shortest at the occipital ROI (contralateral: $144.7 \pm 3.8 \mathrm{~ms}$, ipsilateral: $158.2 \pm 4.5 \mathrm{~ms}$, difference: $13.5 \pm$ $5.4 \mathrm{~ms}$, paired $t$-test, $p<0.05$ ), a few milliseconds slower at the parietal ROI (contralateral: $150.4 \pm 4.3 \mathrm{~ms}$, ipsilateral: $160.3 \pm$ $4.0 \mathrm{~ms}$, difference: $9.9 \pm 4.3 \mathrm{~ms}$, paired $t$-test, $p<0.05)$ and slowest at the temporal ROI (contralateral: $152.5 \pm 4.7 \mathrm{~ms}$, ipsilateral: $175.0 \pm 5.3 \mathrm{~ms}$, difference: $22.5 \pm 6.7 \mathrm{~ms}$, paired $t$-test, $p<0.01)$.

We used these three ROIs (occipital, parietal and temporal), showing stimulation-dependent activations, as potential foci for effects of spatial attention and perceptual awareness in subsequent analyses. The occipital ROI, showing the earliest lateralization effect and the earliest peak latency, may reflect activations in early visual cortex. The later effects observed in the parietal and temporal ROIs may correspond to visual activations in the dorsal and ventral streams, respectively.

\section{ANTICIPATORY ORIENTING OF ATTENTION TOWARD THE CUED LOCATION}

We looked for classical electrophysiological signatures of spatial attention in ongoing neuromagnetic signals before target onset, using the three a priori defined ROIs (see Materials and Methods). We found significant attentional modulations of both eventrelated fields and alpha-band oscillations over parietal cortex before target onset.

The central cue evoked a sustained modulation of neural activity at the parietal ROI from $400 \mathrm{~ms}$ onwards following cue onset, whose spatial pattern depended on the direction of the symbolic spatial cue: cue-evoked responses were stronger contralaterally to the cued location (contralateral minus ipsilateral hemisphere, paired $t$-test, $p<0.01$ ) (Figure 3A). Importantly, this attentional "baseline shift" did not differ whether upcoming targets presented at the cued location were detected or missed (repeated-measures ANOVA, interaction: $p>0.2$ ) (Figure 3B).

Attentional orienting before stimulus onset was further confirmed by the presence of a baseline shift in the alpha frequency band $(9-12 \mathrm{~Hz})$ observed in the same latency range: the orienting of spatial attention induced a focal and sustained suppression of alpha-band activity at the parietal ROI contralateral to the cued location (contralateral minus ipsilateral hemisphere, paired $t$-test, $p<0.001$ ) (Figure 3C), as previously reported (e.g., Worden et al., 2000; Thut et al., 2006; Siegel et al., 2008; Wyart and Tallon-Baudry, 2008, 2009; Gould et al., 2011). As for cueevoked responses, the parietal baseline shift observed in the alpha frequency band was equally strong before detected and missed targets presented at the cued location (repeated-measures ANOVA, hits: $p<0.05$, misses: $p<0.05$, interaction: $p>0.5$ ) (Figure 3D). Furthermore, the amount of prestimulus alpha suppression did not predict by itself whether the upcoming target would be detected or missed (paired $t$-test, $p>0.2$ ).

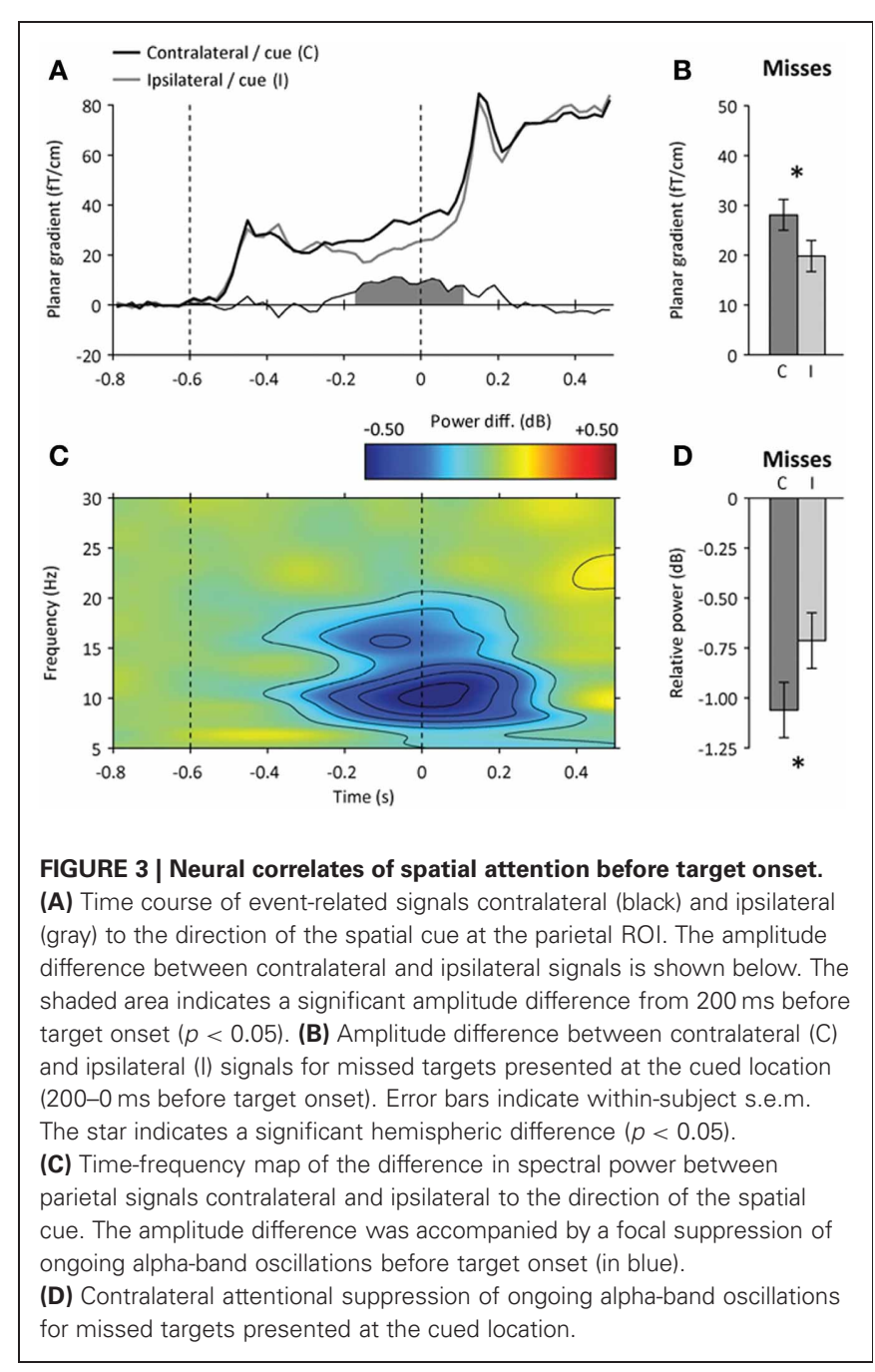

Together, these results show that participants effectively oriented their spatial attention toward the cued location before target onset. Importantly, the strength of this orienting in parietal areas did not differ between detected and missed targets presented at the cued location. Therefore, the prestimulus orienting of spatial attention, as reflected in ongoing modulations of neuromagnetic signals, did not determine by itself the conscious detection of the masked target.

\section{EARLY ATTENTIONAL AMPLIFICATION OF TARGET PROCESSING}

We then assessed whether spatial attention enhanced early visual processing following target onset. Based on previous EEG (Hillyard and Anllo-Vento, 1998; Luck et al., 2000) and MEG reports (Noesselt et al., 2002), we looked for an early attentional modulation of occipital responses around $100 \mathrm{~ms}$ following target onset. We first removed the influence of cue-triggered attentional baseline shifts by using a baseline just before target onset (at 200-0 ms before target onset). This way, any difference between cued and miscued stimuli would reflect attentional modulations that occurred after target onset, and not the anticipatory orienting of attention. As expected, we observed a focal attentional amplification of the contralateral visual response at $90-110 \mathrm{~ms}$ 
following target onset (Figure 4A), peaking at the occipital ROI (main effect: hemisphere, $p<0.005$, interaction: hemisphere $\times$ attention, $p=0.01$, contralateral to target: $p=0.01$, ipsilateral to target: $p>0.2$ ) (Figure 4B). This early attentional amplification was transient, in the sense that it did not reach significance anymore at 110-130 ms following target onset (main effect: hemisphere, $p<0.001$, interaction: hemisphere $\times$ attention, $p>0.1$ ), even though the occipital response was not only stronger, but also more lateralized contralaterally to visual stimulation in this latency range (main effect: time, $p<0.005$, interaction: time $\times$ hemisphere, $p<0.005$ ).

To make sure that this attentional amplification of early occipital responses corresponded to target processing and not to mask processing, we compared the lateralization effects relative to visual stimulation in target-mask and mask-only trials. We reasoned that any lateralized neural activity present in targetmask trials but absent in mask-only trials would reflect target processing, not mask processing. Note that we did not subtract mask-only trials from target-mask trials, but tested whether the early occipital response contralateral to the target and mask was or was not present in mask-only trials at this latency $(90-110 \mathrm{~ms}$ following target onset, i.e., $40-60 \mathrm{~ms}$ following mask onset). We found a significant interaction between the lateralization effects observed in target-mask and mask-only trials (factor "trial type") at 90-125 ms following target onset (repeatedmeasures ANOVA, interaction: hemisphere $\times$ trial type, $p<$ $0.01)$ : no lateralization effect was visible in mask-only trials (post-hoc comparison, $p>0.2$ ). We used a permutation test to verify that this significant interaction was not merely due to the higher number of target-mask trials (corrected $p<0.01$, see Materials and Methods for details). The fact that the lateralization effect was significantly stronger in target-mask trials than in mask-only trials around $100 \mathrm{~ms}$ following target onset thus suggests that the attentional amplification of early visual responses corresponds to target processing and not to mask processing.

By further sorting trials into hits and misses, we then tested whether this early attentional amplification critically differed depending on participants' awareness of the target. This was not the case (repeated-measures ANOVA, interaction: awareness $\times$
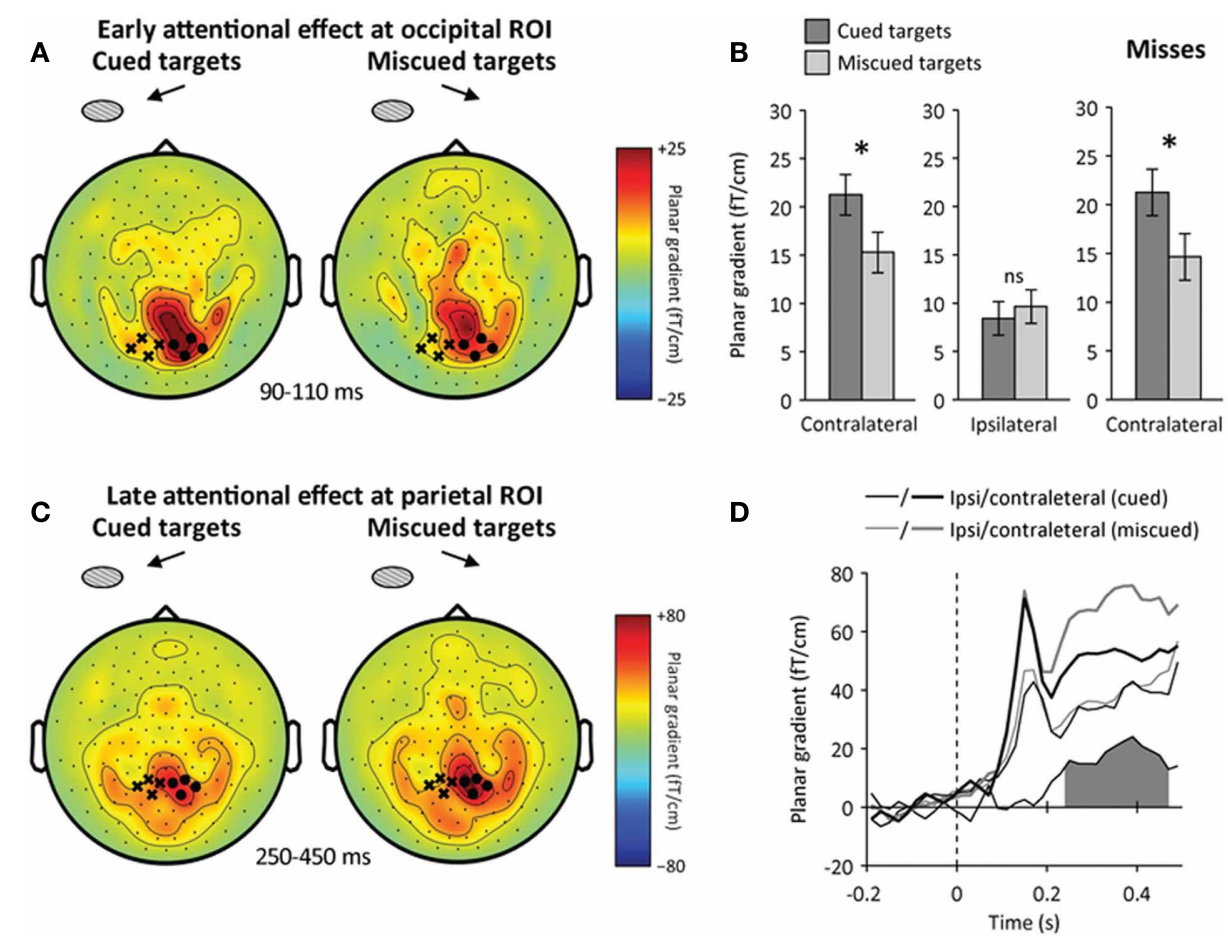

FIGURE 4 | Neural correlates of spatial attention following target onset. (A) Early attentional enhancement at occipital ROI. Sensor-level topography of early neuromagnetic event-related signals at $90-110 \mathrm{~ms}$ following target onset (left: cued targets, right: miscued targets). The contralateral (ipsilateral) occipital $\mathrm{ROI}$ is indicated by black dots (cross, respectively). The contralateral occipital response was increased for cued relative to miscued targets.

(B) Early attentional amplification of the contralateral occipital response to cued (dark) relative to miscued (light) targets. Error bars indicate within-subject s.e.m. A star indicates a significant effect $(p<0.05)$, ns a non-significant effect. Spatial attention enhanced the contralateral (left), but not the ipsilateral (center) occipital response. Right: spatial attention significantly enhanced the contralateral occipital response to missed targets.
(C) Late attentional reorienting at parietal ROI. Sensor-level topography of late neuromagnetic signals at 250-450 ms following target onset (left: cued, right: miscued). The contralateral (ipsilateral) parietal ROI is indicated by black dots (crosses, respectively). Contralateral parietal signals were increased for miscued relative to cued trials, reflecting the reorienting of spatial attention toward masks presented at the uncued location. (D) Time course of the reorienting effect at the parietal $\mathrm{ROI}$ (black: cued, gray: miscued, thick: contralateral to stimulation, thin: ipsilateral to stimulation). The difference between miscued and cued contralateral parietal signals is shown below. The shaded area indicates a significant difference $(p<0.05)$. The reorienting effect was sustained at the contralateral parietal ROI at $250-450$ ms relative to target onset. 
attention, $p>0.5)$. Critically, the early attentional amplification was observed even when the target was missed (post-hoc comparison, $p=0.01$ ) (Figure 4B). Furthermore, the amplitude of the contralateral occipital response in this latency range did not predict by itself whether the target would be detected (main effect: awareness, $p>0.5$ ).

Therefore, the early attentional amplification of occipital responses around $100 \mathrm{~ms}$ was identical for detected and missed targets: this early attentional amplification was observed even when the target was not consciously detected, and did not increase the probability of consciously detecting the target. These results, therefore, suggest that the early amplification of visual signals by spatial attention did not control conscious access to the masked targets, in accordance with the behavioral observation that spatial attention did not increase the proportion of detected targets at the cued location.

\section{LATE REORIENTING OF ATTENTION TOWARD UNCUED STIMULI}

We also observed a second, late difference between the neuromagnetic responses to cued and miscued stimuli at the parietal ROI (Figure 4C). A closer inspection of this difference suggested it corresponded to the reorienting of spatial attention toward stimuli presented at the uncued location (which were clearly signaled by the unilateral and high-contrast mask). Indeed, the contralateral parietal activity was focally increased in response to miscued stimuli, relative to cued ones (repeated-measures ANOVA, interaction: hemisphere $\times$ attention, $p<0.01$ ) (Figure 4D). We observed an amplification of contralateral responses to miscued stimuli at the parietal and temporal ROIs at 220-450 ms following target onset (paired $t$-test, parietal ROI: $p<0.005$, temporal ROI: $p<0.01)$. Furthermore, as it was the case before target onset, the increased parietal activity was accompanied by a focal suppression of alpha-band activity contralateral to miscued stimuli in the same time window (paired $t$-test, $p<0.005$ ).

Together, these three successive cue-dependent effects reveal the spatiotemporal dynamics of endogenous spatial attention over the course of a trial: (1) an anticipatory orienting toward the cued location before target onset, (2) an early amplification of target processing occurring focally in time around $100 \mathrm{~ms}$ following target onset (Figure 6B), and (3) a late reorienting toward stimuli presented at the uncued location.

\section{EARLIEST NEUROMAGNETIC CORRELATE OF CONSCIOUS DETECTION}

We looked for neuromagnetic correlates of conscious detection at the occipital, parietal, and temporal ROIs by computing the time course of the statistical difference between lateralization effects to detected and missed targets. The earliest neuromagnetic correlate of conscious detection was observed at 110-130 ms following target onset, slightly later than the early attentional amplification of target processing (Figure 5A, left). In this latency range, contralateral visual responses were significantly stronger when participants detected the masked target than when they missed it. Contralateral neuromagnetic responses differed between hits and misses slightly at the occipital ROI (repeated-measures ANOVA, interaction: hemisphere $\times$ awareness, $p<0.05$, contralateral hemisphere: $p=0.05)$, more strongly at the temporal ROI (repeated-measures ANOVA, interaction: hemisphere $\times$ awareness, $p<0.001$, contralateral hemisphere: $p<0.05$ ), and did not differ at the parietal ROI (repeated-measures ANOVA, interaction: hemisphere $\times$ awareness, $p>0.1$, contralateral hemisphere: $p>0.5$ ) (Figure 5A, right). This early correlate of conscious detection was thus spatially confined to contralateral occipito-temporal ROI, before the peak of the maskdriven visual activation observed at $150 \mathrm{~ms}$ following target onset (Figure 5B, left, and Figure 6A).

We then tested whether this early correlate of conscious detection depended on the locus of attention (i.e., the cued location). The effect was significant both when the masked target was presented in the focus of attention (repeated-measures ANOVA, interaction: hemisphere $\times$ awareness, $p<0.05$, contralateral: $p<$ 0.05 ) or away from the focus of attention (repeated-measures ANOVA, interaction: hemisphere $\times$ awareness, $p<0.05$, contralateral: $p<0.005$ ) (Figure 5C). Furthermore, the amplitude of the contralateral temporal activation was not significantly modulated by the locus of attention in this latency range (repeated-measures ANOVA, interaction: hemisphere $\times$ attention, $p>0.1$, contralateral: $p>0.1$ ). This early awarenessdependent activation thus appeared relatively independent from the locus of spatial attention.

In addition to this early difference between hits and misses (i.e., identical physical stimulation, but different subjective perception), a slight amplitude difference between misses and correct rejects (i.e., different physical stimulation, but same subjective perception) was observed in the same latency range at the occipital ROI (paired $t$-test, $p<0.01$ ). Besides this amplitude difference between hits, misses, and correct rejects, the peak latency over the temporal region also gradually differed between hits, misses, and correct rejects (hits: $145.1 \pm 4.5 \mathrm{~ms}$, misses: $154.5 \pm 5.1 \mathrm{~ms}$, difference with hits: $9.3 \pm 3.5 \mathrm{~ms}, p<0.05$; correct rejects: $164.1 \pm 4.5 \mathrm{~ms}$, difference with misses: $9.6 \pm 4.2 \mathrm{~ms}$, $p<0.05$ ) (Figure 5B, right). These additional results indicate the presence of target processing even on missed trials, when participants did not consciously detect the presence of a target. In that sense, the early occipital and temporal activations correlated with both objective physical information and subjective perception.

\section{LATE NEUROMAGNETIC SIGNATURE OF CONSCIOUS REPORTABILITY}

Following the peak of the mask-driven visual response, around $150 \mathrm{~ms}$ following target onset, we observed a second correlate of conscious detection in the sustained part of the contralateral visual response from $220 \mathrm{~ms}$ onwards following target onset at the temporal ROI (repeated-measures ANOVA, main effect: awareness, $p=0.01$ ) (Figure 5B, left). No significant differences were observed at the occipital and parietal ROIs. In addition to being significant at the contralateral temporal ROI, this late and sustained awareness-dependent activation differed from the early effect in the sense that it was distributed bilaterally, with significant differences between hits and misses at the ipsilateral temporal ROI as well (interaction: hemisphere $\times$ awareness, $p>$ 0.2 , ipsilateral hemisphere: $p<0.05$ ). Last, a difference between hits and misses was found at frontal sensors that were not defined a priori (paired $t$-test, $p<0.05$ ) (Figure 5D).

In this latency range, responses to misses and correct rejects were no longer different, even at the occipital ROI where 


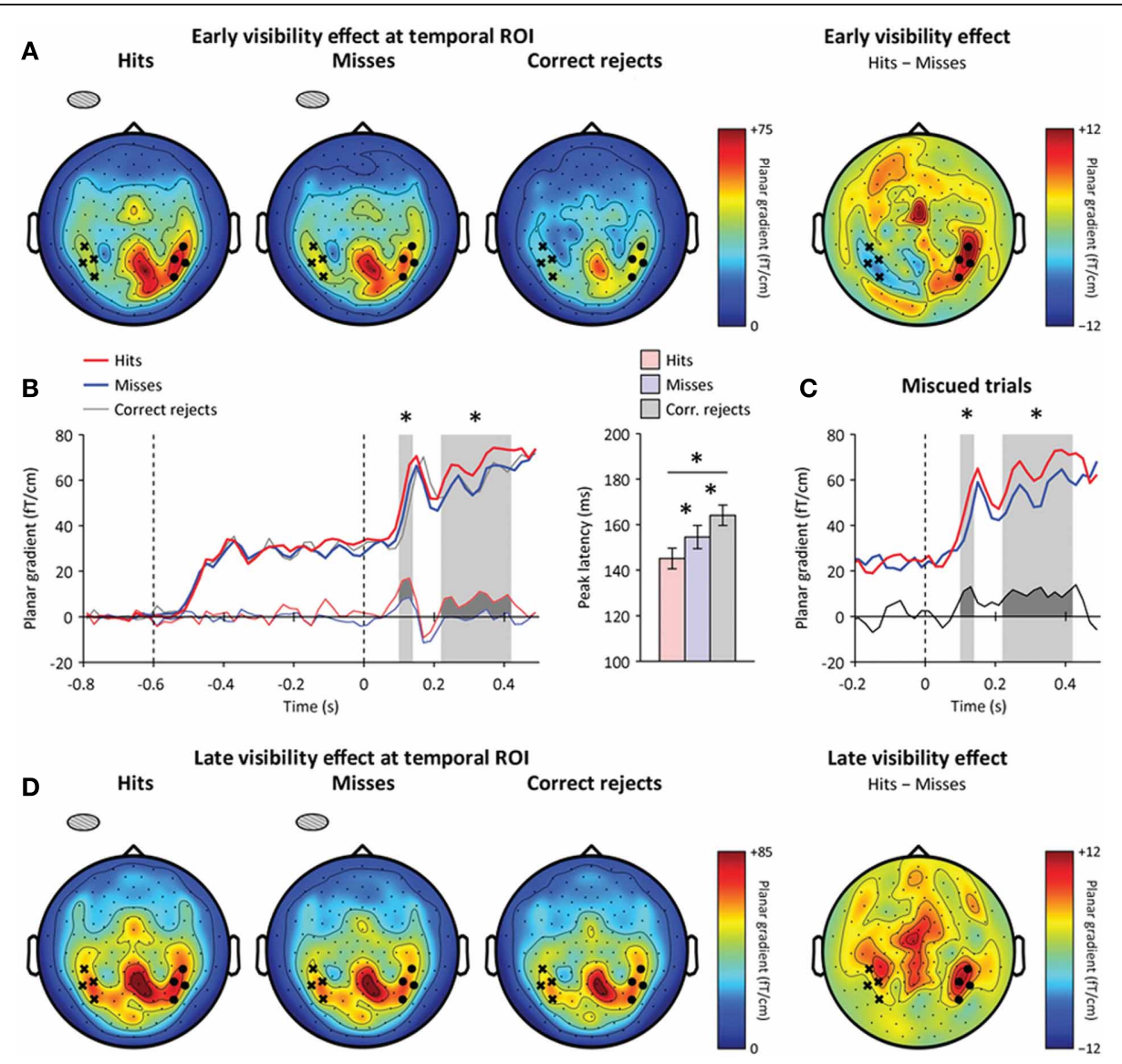

FIGURE 5 | Neural correlates of conscious detection. (A) Early awareness-dependent effect. Left: Sensor-level topography of neuromagnetic event-related signals at $110-130 \mathrm{~ms}$ following target onset (left: hits, center: misses, right: correct rejects). The contralateral (ipsilateral) temporal ROI is highlighted by black dots (crosses, respectively). Right: Sensor-level topography of the awareness-dependent effect (hits minus misses). The early awareness-dependent effect peaks at the contralateral temporal ROI. (B) Left: Time course of contralateral signals at the temporal ROI for hits (red), misses (blue), and correct rejects (gray). The thin red (blue) lines below indicate the amplitude difference between hits (misses) and correct rejects (respectively).
Stars indicate significant differences between hits and misses $(p<0.05)$. Right: Peak latencies at the contralateral temporal ROI for hits, misses and correct rejects. Peak latencies differed both between hits and misses, and between misses and correct rejects. Error bars indicate within-subject s.e.m. (C) Time course of contralateral temporal signals for miscued trials only: similar awareness-dependent effects were observed. The difference between hits and misses is shown below. (D) Left: Sensor-level topography of late signals at $220-420 \mathrm{~ms}$ following target onset. Right: sensor-level topography of the late awareness-dependent effect (hits minus misses). The late awareness-dependent effect peaks at bilateral temporal and frontal sensors. a significant difference was observed before the peak of the mask-driven visual response (paired $t$-test, $p>0.2$ ) (Figure 5D). Therefore, this late correlate of conscious detection appeared "all-or-none," in the sense that the distributed neuromagnetic activation observed in this latency range reflected subjective perception (i.e., a significant difference between hits and misses), but not physical stimulation (i.e., no difference between misses minus correct rejects). In that sense, this late, sustained awareness-dependent effect was qualitatively different from the early, transient effect which reflected both objective physical stimulation and subjective perception of the masked target.

\section{LATE INTERACTION BETWEEN SPATIAL ATTENTION AND CONSCIOUS DETECTION}

To what extent did this late awareness-dependent effect depend on the locus of attention? We first found that the amplitude difference between hits and misses was present for both cued and miscued stimuli (repeated-measures ANOVA, interaction: attention $\times$ awareness: $p>0.2$, cued: $p<0.05$, miscued: $p<$ 0.05) (Figure 5C). This result could suggest that the late awareness-dependent activation is independent from endogenous spatial attention.

However, in this latency range, the high-contrast masks presented at the uncued location have already triggered a reorienting of attention (Figure 4D), which was found to differ between detected and missed targets presented at the uncued location (Figure 7A). At the parietal ROI, the neuromagnetic signature of the reorienting of attention emerged earlier when the target was consciously detected (Figure 7B, left): at 220-250 ms following target onset, the contralateral parietal activation to miscued stimuli was stronger for hits than for misses (paired $t$-test, $p<0.05$ ). Then, at the temporal ROI, the sustained part of the reorienting of attention was stronger for hits than for misses at $250-450 \mathrm{~ms}$ 


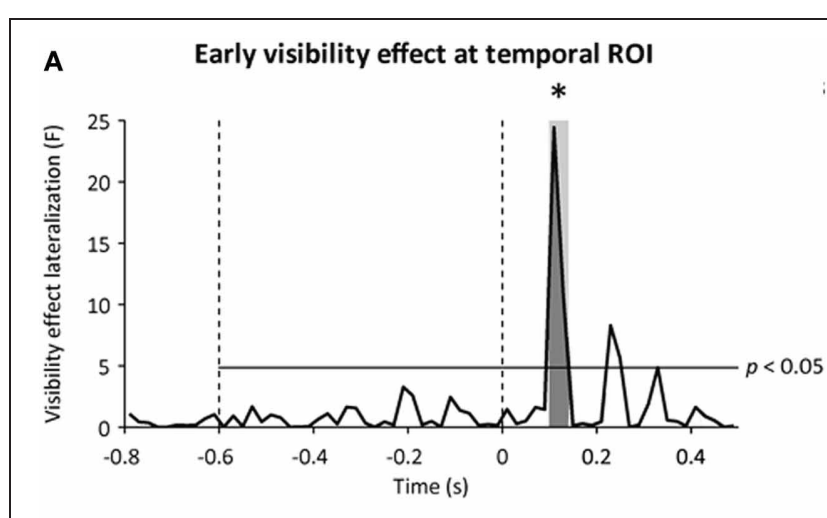

B Early attentional effect at occipital ROI
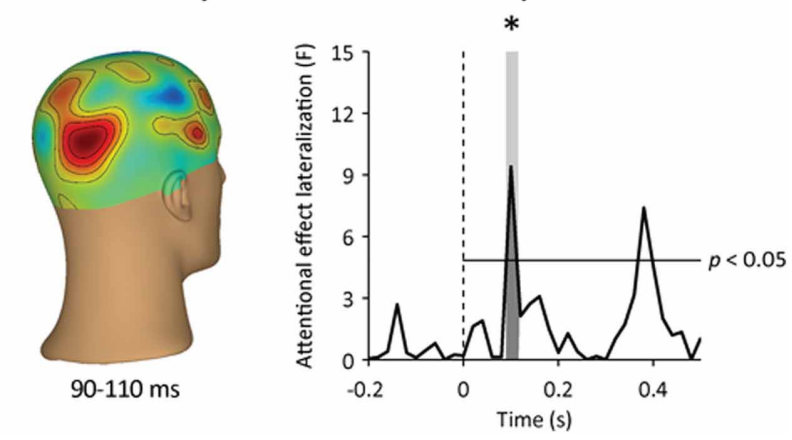

FIGURE 6 | Time courses of awareness- and attention-dependent effects. (A) Time course of the awareness-dependent effect at the temporal ROI. The visibility effect lateralization corresponds to the lateralization of the awareness-dependent effect (hits minus misses) in the event-related MEG response to visual stimulation at the temporal $\mathrm{ROI}$ expressed in $\mathrm{F}$ units. The earliest significant awareness-dependent effect occurred at 110-130 ms following target onset. (B) Time course of the attention-dependent effect at the occipital ROI. Left: three-dimensional topography of the early attention-dependent effect (valid minus invalid targets) at 90-110 ms following target onset. Right: the attentional effect lateralization corresponds to the lateralization of the attention-dependent effect (valid minus invalid targets) in the event-related MEG response to visual stimulation at the occipital $\mathrm{ROI}$, expressed in $\mathrm{F}$ units. The earliest significant attention-dependent effect occurred at 90-110 ms following target onset. Horizontal lines indicate an uncorrected statistical threshold at $p<0.05$. Stars indicate significant awareness- and attention-dependent effects $(p<0.05)$.

following target onset (repeated-measures ANOVA, interaction: attention $\times$ awareness: $p<0.05$, hits: $p<0.01$, misses: $p>0.2$ ) (Figure 7B, right). This pattern of results suggests that miscued targets triggered an earlier and stronger reorienting of attention when the target was consciously detected than when it was missed.

\section{DISCUSSION}

The present results reveal an early dissociation between endogenous spatial attention and perceptual awareness during visual masking. Spatial attention increased the amplitude of occipital electromagnetic responses at $100 \mathrm{~ms}$ following target onset. However, this attentional amplification of early visual processing was found to be identical for detected and missed targets. This result not only provides additional neural evidence for attentional modulations of non-conscious processing (Naccache et al., 2002; Woodman and Luck, 2003; Kiefer and Brendel, 2006; Koivisto et al., 2006; Koivisto and Revonsuo, 2007, 2008), but further suggests that early attentional amplification does not have a decisive impact on the conscious detection of a single, masked target. The first neuromagnetic correlate of conscious detection was observed $20 \mathrm{~ms}$ later and corresponded to earlier and stronger activations for detected than missed targets in ventral visual cortex, independently from the locus of spatial attention. These results, therefore, further suggest that the neural amplification thought to be necessary for a stimulus to reach consciousness (Dehaene et al., 2006) does not necessarily depend on endogenous spatial attention.

Following the peak of the mask-driven visual activation, a second neuromagnetic signature of conscious perception emerged bilaterally from $220 \mathrm{~ms}$ in temporal cortex, and extended to frontal cortex. This late awareness-dependent effect coincided in time with the late reorienting of spatial attention toward the highcontrast masks presented at the uncued location. The latency and amplitude of this reorienting effect depended on whether the preceding target was consciously detected or missed, suggesting an influence of conscious access on the capture of attention - an effect whose direction runs opposite to the usual view according to which spatial attention controls conscious access (e.g., Posner, 1994).

Our results, therefore, confirm the double dissociation between the neural correlates of endogenous spatial attention and visual awareness (Schurger et al., 2008; Wyart and TallonBaudry, 2008), and further show that this dissociation can be found even at early stages of visual processing during visual masking. However, because we focused our analyses on lateralized brain signals corresponding to processing in retinotopically organized visual areas, our results do not rule out the possibility of additional, non-lateralized correlates of both attention and awareness.

\section{ORIENTING OF ATTENTION AND CONSCIOUS DETECTION}

The present MEG data clearly indicate that subjects oriented their attention according to the central cue. Before target onset, we observed a sustained attentional modulation of ongoing signals in the contralateral parietal cortex from $400 \mathrm{~ms}$ following cue onset, which was accompanied by a focal suppression of alphaband oscillations in the same region. Both effects have previously been related to the goal-directed orienting of attention in space (Thut et al., 2006; Siegel et al., 2008; Wyart and Tallon-Baudry, 2008, 2009; Gould et al., 2011). Furthermore, following target onset, spatial attention increased the amplitude of early visual responses to cued targets at about $100 \mathrm{~ms}$ following target onset, thereby matching numerous previous observations (Hillyard and Anllo-Vento, 1998; Hillyard et al., 1998; Luck et al., 2000; Noesselt et al., 2002). These two successive neuromagnetic effects indicate that subjects effectively oriented their attention toward the cued location. It is important to note that these two successive effects are functionally distinct: while attention increases the evoked response for targets presented contralaterally to the cued location, attention concurrently suppresses alpha-band activity in the $500 \mathrm{~ms}$ surrounding target onset. If the observed alpha-band 


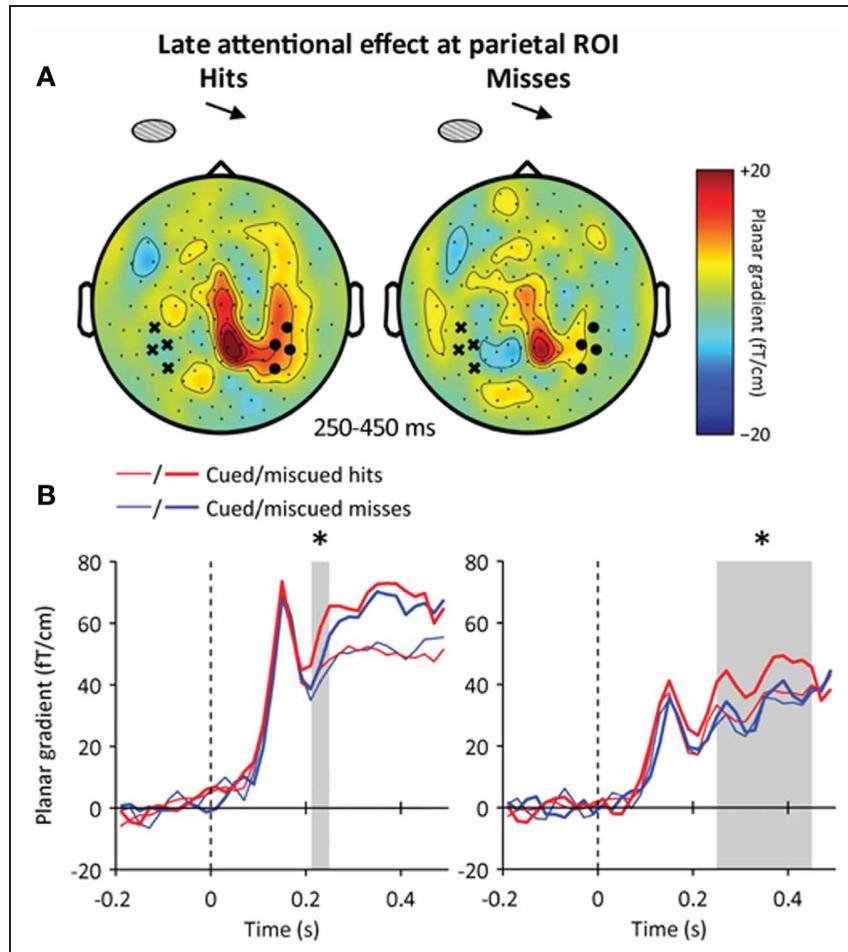

FIGURE 7 | Late interaction between spatial attention and conscious detection. (A) Sensor-level topography of the late reorienting effect (miscued minus cued trials) for hits (left) and misses (right) at 250-450 ms following target onset. The contralateral (ipsilateral) temporal ROI is indicated by black dots (crosses, respectively). The reorienting effect at the temporal ROI was stronger when the target was detected than missed. (B) Time course of the reorienting effect for hits (red) and misses (blue) at the parietal (left) and temporal (right) ROls. The reorienting effect started 30 ms earlier for hits than misses at the parietal ROI, and was stronger for hits than misses at the temporal ROI. Stars indicate significant effects $(p<0.05)$.

suppression was an artifact of the attentional modulation of target processing, there should be an enhancement (rather than a suppression) of alpha power contralateral to the cued location around target onset.

Surprisingly, the strength of this attentional orienting, both preceding and following target onset, did not determine the detectability of the masked target, since the proportion of consciously detected targets did not increase at the cued location. Furthermore, retrospective analyses showed that the strength of the attentional modulation of ongoing parietal signals did not differ between detected and missed targets presented at the cued location, indicating that participants did not miss the presence of the masked target because they did not orient their attention at the cued location.

The fact that spatial attention did not have any significant effect on the conscious detection of the masked gratings might appear surprising, since spatial attention has previously been shown to increase perceptual performance in masking conditions (Smith et al., 2004; Smith and Ratcliff, 2009). However, a number of differences across studies can explain this apparent discrepancy. First, we used metacontrast masks, surrounding without overlapping the target location, which are known to have different masking properties than regular pattern masks (Breitmeyer and Öğmen, 2006; Ishikawa et al., 2006). Critically, the subjective appearance of the target also differed across studies: in the present study, participants could detect the presence of the metacontrast-masked target without being able to coarsely discriminate its orientation better than chance (see also Vorberg et al., 2003), while the conscious detection of the pattern-masked target was reported to be as easy as the coarse discrimination of its orientation in previous studies (Smith et al., 2004). These results thus suggest that the effect of spatial attention on conscious detection is smaller when the conscious detection of the target is dissociated from the identification of its physical properties, at least in masking conditions. Furthermore, the present study manipulated sustained spatial attention using informative central cues, whereas previous studies manipulated transient spatial attention using non-informative peripheral cues (e.g., Smith et al., 2004). It was recently suggested that while exogenous attention facilitates conscious detection, this may not be systematically the case for endogenous attention (Chica et al., 2011). Besides, the use of a unilateral mask suppressed spatial uncertainty, which is known to strongly reduce attentional benefits for unmasked targets (Gould et al., 2007). Because all the known neuromagnetic markers of endogenous attention are present in our study (ongoing alpha-band suppression in response to the cue, increased amplitude of early phase-locked responses to the stimulus), the absence of behavioral influence of attention on conscious detection suggests that in the specific experimental conditions studied here, spatial attention does not facilitate conscious detection (Herrmann et al., 2010). This parsimonious interpretation would explain both the behavioral results and the observation that the early neural correlates of spatial attention and visual awareness were independent. The absence of attentional effect on behavior might also be explained by the fact that we used delayed tasks with variable response mapping, in contrast to most tasks which asked for speeded responses with a fixed response mapping. The use of this delayed responding scheme was crucial to distinguish perceptual effects from late decision or motor effects (Wyart and Tallon-Baudry, 2008), but it is not unsurprising that in these delayed responding conditions, spatial attention did not have a strong behavioral effect on reaction times.

\section{EARLY DISSOCIATION BETWEEN SPATIAL ATTENTION AND CONSCIOUS DETECTION}

The present results reveal an early dissociation between the neuromagnetic correlates of spatial attention and of conscious detection: before the onset of mask-driven responses, spatial attention increased the amplitude of early occipital activations both to detected and missed targets at $100 \mathrm{~ms}$ following target onset, whereas the earliest neuromagnetic correlate of conscious detection was observed only $20 \mathrm{~ms}$ later in the ventral visual cortex, independently from the locus of attention.

These results provide additional evidence for the existence of early neural correlates of consciousness. The first EEG description of such an effect, observed at $100 \mathrm{~ms}$ following stimulus onset (Pins and Ffytche, 2003), remains debated since the reported 
effect was weak, not always replicated (Koivisto et al., 2008), and potentially due to uncontrolled attentional modulations of early visual processing. The finer spatial resolution of MEG recordings allowed us to report a robust, retinotopically-constrained correlate of consciousness at early latencies (Boehler et al., 2008), and crucially to dissociate this effect from the attentional amplification of early visual processing. We, therefore, extend previous observations (Schurger et al., 2008; Wyart and Tallon-Baudry, 2008), by showing that within the same trials, when attention and awareness are simultaneously manipulated and measured, the neural correlates of endogenous spatial attention and visual awareness can be distinct as early as $100 \mathrm{~ms}$ following stimulus onset. This suggests that the early neural amplification of attended stimuli does not necessarily have a causal role in conscious access - at least in the experimental conditions tested here (central predictive cues, metacontrast masks, no competition between stimuli).

Importantly, in our study, the first attentional effect that reached significance occurred just before $100 \mathrm{~ms}$. We did not find an earlier attentional effect around $75 \mathrm{~ms}$ (Poghosyan and Ioannides, 2008), maybe because the gratings we used were not presented at $100 \%$ contrast for hundreds of milliseconds, but at $15 \%$ contrast and for only $17 \mathrm{~ms}$ because we wanted them to be at detection threshold-i.e., undetected on half of the trials. This could explain why earlier visual responses, that can potentially be modulated by attention, may not have been strong enough to be detected. Most visual areas beyond V1/V2 are already active at $100 \mathrm{~ms}$ following the onset of a visual stimulus (Noesselt et al., 2002), and we, therefore, cannot determine the precise localization of the observed attentional enhancement of visual responses at $90-110 \mathrm{~ms}$, except that it occurs in retinotopically organized visual areas.

The early attentional amplification observed for both detected and missed targets was not accompanied by a reduction in response latency, as already described in similar conditions (e.g., Mangun, 1995; Luck et al., 2000). Therefore, this amplification cannot be attributed to a simple contrast enhancement of attended stimuli (Lee et al., 2007). By contrast, the early awareness-dependent effect included a significant reduction in response latency to detected targets. This result is not surprising, since the visual information about target presence is strongly latency-dependent during visual masking. Indeed, the neural response to a masked target is known to be rapidly interrupted by the neural response to the following mask (Breitmeyer and Öğmen, 2006): the initial, early response to the target thus becomes highly informative to detect its presence (Thorpe et al., 1996; VanRullen and Thorpe, 2001, 2002).

\section{THE PROGRESSIVE BUILD-UP OF CONSCIOUS ACCESS}

The early correlate of consciousness was observed before maskdriven visual activations and was confined to contralateral visual cortex (Boehler et al., 2008), whereas the second, late correlate of consciousness emerged after the peak of mask-driven visual activations in bilateral temporal (Koivisto et al., 2008) and frontal cortices (Dehaene et al., 2001; Del Cul et al., 2007). Both the early and late correlates of consciousness were independent from the endogenous, cue-triggered orienting of attention. However, they differed qualitatively, in the sense that early contralateral activations in temporal cortex did not constitute a "pure" reflection of subjective conscious perception, since they also depended on the presence of objective physical stimulation (larger and earlier responses for hits than for misses, but also for misses than for correct rejects). In contrast, the late neural signature of consciousness was all-or-none, in the sense that it correlated exclusively with conscious perception: stronger activations for detected than for missed targets, but identical activations for missed and physically-absent targets. Similar results have recently been reported using intracranial recordings (Fisch et al., 2009).

The fact that the early correlate of consciousness was graded, while the second was all-or-none suggests that the two reflect qualitatively-distinct processes during the buildup of the final phenomenal percept of the masked target. The first, transient correlate may reflect a perceptual modulation that increases the probability of subsequent conscious reportability (e.g., Pins and Ffytche, 2003; Boehler et al., 2008; Koivisto et al., 2008), while the second, sustained correlate over temporal and frontal cortices may correspond to a perceptual decision stage closer to subjective perception (Beck et al., 2001; Marois et al., 2004; Del Cul et al., 2007). This comprehensive view of the time course of the neural correlates of consciousness could potentially reconcile early and late accounts of conscious access: the sensory evidence upon which conscious detection depends arises from processes taking place at both early and late stages, and even before stimulus onset (Boly et al., 2007; Hesselmann et al., 2008; van Dijk et al., 2008; Wyart and Tallon-Baudry, 2009). Whether or not a stimulus ultimately becomes conscious would depend on the cumulative contribution of these multiple sensory influences, whose sum ultimately leads to a subjective decision reflected in late (>200 ms) electromagnetic recordings (Del Cul et al., 2007).

To conclude, our results confirm the double dissociation between the neural correlates of endogenous spatial attention and visual awareness found in healthy human subjects (Schurger et al., 2008; Wyart and Tallon-Baudry, 2008), and further show that this dissociation can be found even at early stages of visual processing during visual masking. However, if the attentional amplification of early visual processing does not seem to have a decisive impact on conscious reportability, it does not follow that all attentional mechanisms are unrelated to perceptual awareness (see, e.g., Sergent et al., 2005). In cluttered displays, attentional processes rely not only on target amplification, but more critically on the competition between the target and the surrounding distractors (Desimone and Duncan, 1995; Reynolds and Chelazzi, 2004): under high load, these competition mechanisms are thought to be responsible for the striking capacity limits of conscious reportability (Simons and Chabris, 1999).

\section{ACKNOWLEDGMENTS}

This work was supported by grants from Région Ile-de-France to Valentin Wyart and Agence Nationale de la Recherche to Catherine Tallon-Baudry. We are grateful to A. Ducorps, D. Schwartz, and J. D. Lemaréchal for technical assistance. 


\section{REFERENCES}

Baars, B. J. (1988). A Cognitive Theory of Consciousness. Cambridge: Cambridge University Press.

Bashinski, H. S., and Bacharach, V. R. (1980). Enhancement of perceptual sensitivity as the result of selectively attending to spatial locations. Percept. Psychophys. 28, 241-248.

Bastiaansen, M. C., and Knosche, T. R. (2000). Tangential derivative mapping of axial MEG applied to eventrelated desynchronization research. Clin. Neurophysiol. 111, 1300-1305.

Bauer, M., Oostenveld, R., Peeters, M., and Fries, P. (2006). Tactile spatial attention enhances gammaband activity in somatosensory cortex and reduces low-frequency activity in parieto-occipital areas. J. Neurosci. 26, 490-501.

Beck, D. M., Rees, G., Frith, C. D., and Lavie, N. (2001). Neural correlates of change detection and change blindness. Nat. Neurosci. 4, 645-650.

Boehler, C. N., Schoenfeld, M. A., Heinze, H. J., and Hopf, J. M. (2008). Rapid recurrent processing gates awareness in primary visual cortex. Proc. Natl. Acad. Sci. U.S.A. 105, 8742-8747.

Boly, M., Balteau, E., Schnakers, C., Degueldre, C., Moonen, G., Luxen, A., Phillips, C., Peigneux, P., Maquet, P., and Laureys, S. (2007). Baseline brain activity fluctuations predict somatosensory perception in humans. Proc. Natl. Acad. Sci. U.S.A. 104, 12187-12192.

Brainard, D. H. (1997). The Psychophysics Toolbox. Spat. Vis. 10, 433-436.

Breitmeyer, B. G., and Öğmen, H. (2006). Visual Masking: Time Slices Through Conscious and Unconscious Vision. Oxford, UK: Oxford University Press.

Carrasco, M. (2011). Visual attention: the past 25 years. Vision Res. 51, 1484-1525.

Carrasco, M., Ling, S., and Read, S. (2004). Attention alters appearance. Nat. Neurosci. 7, 308-313.

Chica, A. B., Lasaponara, S., Chanes, L., Valero-Cabre, A., Doricchi, F., Lupianez, J., and Bartolomeo, P. (2011). Spatial attention and conscious perception: the role of endogenous and exogenous orienting. Atten. Percept. Psychophys. 73, 1065-1081.

Dehaene, S., Changeux, J. P., Naccache, L., Sackur, J., and Sergent, C. (2006). Conscious, preconscious, and subliminal processing: a testable taxonomy. Trends Cogn. Sci. 10, 204-211.

Dehaene, S., Naccache, L., Cohen, L., Bihan, D. L., Mangin, J. F., Poline, J.
B., and Riviere, D. (2001). Cerebral mechanisms of word masking and unconscious repetition priming. Nat. Neurosci. 4, 752-758.

Del Cul, A., Baillet, S., and Dehaene, S. (2007). Brain dynamics underlying the nonlinear threshold for access to consciousness. PLoS Biol. 5, e260. doi: 10.1371/journal.pbio.0050260

Dennett, D. (1991). Consciousness Explained. Boston, MA: Little and Brown.

Desimone, R., and Duncan, J. (1995). Neural mechanisms of selective visual attention. Annu. Rev. Neurosci. 18, 193-222.

Faivre, N., and Kouider, S. (2011). Multi-feature objects elicit nonconscious priming despite crowding. J. Vis. 11, 3.

Fisch, L., Privman, E., Ramot, M., Harel, M., Nir, Y., Kipervasser, S., Andelman, F., Neufeld, M. Y., Kramer, U., Fried, I., and Malach, R. (2009). Neural “ignition": enhanced activation linked to perceptual awareness in human ventral stream visual cortex. Neuron 64, 562-574.

Gould, I. C., Rushworth, M. F., and Nobre, A. C. (2011). Indexing the graded allocation of visuospatial attention using anticipatory alpha oscillations. J. Neurophysiol. 105, 1318-1326.

Gould, I. C., Wolfgang, B. J., and Smith, P. L. (2007). Spatial uncertainty explains exogenous and endogenous attentional cuing effects in visual signal detection. J. Vis. 7, 4.1-4.17.

Herrmann, K., Montaser-Kouhsari, L., Carrasco, M., and Heeger, D. J. (2010). When size matters: attention affects performance by contrast or response gain. Nat. Neurosci. 13, 1554-1559.

Hesselmann, G., Kell, C. A., and Kleinschmidt, A. (2008). Ongoing activity fluctuations in hMT+ bias the perception of coherent visual motion. J. Neurosci. 28, 14481-14485.

Hillyard, S. A., and Anllo-Vento, L. (1998). Event-related brain potentials in the study of visual selective attention. Proc. Natl. Acad. Sci. U.S.A. 95, 781-787.

Hillyard, S. A., Vogel, E. K., and Luck, S. J. (1998). Sensory gain control (amplification) as a mechanism of selective attention: electrophysiological and neuroimaging evidence. Philos. Trans. R. Soc. Lond. B Biol. Sci. 353, 1257-1270.

Ishikawa, A., Shimegi, S., and Sato, H. (2006). Metacontrast masking suggests interaction between visual pathways with different spatial and temporal properties. Vision Res. 46, 2130-2138.
Jokisch, D., and Jensen, O. (2007). Modulation of gamma and alpha activity during a working memory task engaging the dorsal or ventral stream. J. Neurosci. 27, 3244-3251.

Kentridge, R. W., Heywood, C. A. and Weiskrantz, L. (2004). Spatial attention speeds discrimination without awareness in blindsight. Neuropsychologia 42, 831-835.

Kentridge, R. W., Nijboer, T. C., and Heywood, C. A. (2008). Attended but unseen: visual attention is not sufficient for visual awareness. Neuropsychologia 46, 864-869.

Kiebel, S. J., Tallon-Baudry, C., and Friston, K. J. (2005). Parametric analysis of oscillatory activity as measured with EEG/MEG. Hum. Brain Mapp. 26, 170-177.

Kiefer, M., and Brendel, D. (2006) Attentional modulation of unconscious "automatic" processes: evidence from event-related potentials in a masked priming paradigm. J. Cogn. Neurosci. 18 , 184-198.

Kiefer, M., and Martens, U. (2010) Attentional sensitization of unconscious cognition: task sets modulate subsequent masked semantic priming. J. Exp. Psychol. Gen. 139, 464-489.

Koch, C., and Tsuchiya, N. (2007) Attention and consciousness: two distinct brain processes. Trends Cogn. Sci. 11, 16-22.

Koivisto, M., and Revonsuo, A. (2007) Electrophysiological correlates of visual consciousness and selective attention. Neuroreport 18 753-756.

Koivisto, M., and Revonsuo, A. (2008) The role of selective attention in visual awareness of stimulus features: electrophysiological studies. Cogn. Affect. Behav. Neurosci. 8, 195-210.

Koivisto, M., Revonsuo, A., and Lehtonen, M. (2006). Independence of visual awareness from the scope of attention: an electrophysiological study. Cereb. Cortex 16, 415-424.

Koivisto, M., Lahteenmaki, M., Sorensen, T. A., Vangkilde, S., Overgaard, M., and Revonsuo, A. (2008). The earliest electrophysiological correlate of visual awareness? Brain Cogn. 66, 91-103.

Lamme, V. A. (2003). Why visual attention and awareness are different. Trends Cogn. Sci. 7, 12-18.

Lamme, V. A. (2004). Separate neural definitions of visual consciousness and visual attention; a case for phenomenal awareness. Neural Netw. 17, 861-872.

Lee, J., Williford, T., and Maunsell, J. H. (2007). Spatial attention and the latency of neuronal responses in macaque area V4. J. Neurosci. 27, 9632-9637

Liu, T., Abrams, J., and Carrasco, M. (2009). Voluntary attention enhances contrast appearance. Psychol. Sci. 20, 354-362.

Luck, S. J., Woodman, G. F., and Vogel, E. K. (2000). Event-related potential studies of attention. Trends Cogn. Sci. 4, 432-440.

Mangun, G. R. (1995). Neural mechanisms of visual selective attention. Psychophysiology 32, 4-18.

Marois, R., Yi, D. J., and Chun, M. M. (2004). The neural fate of consciously perceived and missed events in the attentional blink. Neuron 41, 465-472.

Martens, U., Ansorge, U., and Kiefer, M. (2011). Controlling the unconscious: attentional task sets modulate subliminal semantic and visuomotor processes differentially. Psychol. Sci. 22, 282-291.

Martinez-Trujillo, J., and Treue, S. (2002). Attentional modulation strength in cortical area MT depends on stimulus contrast. Neuron 35, 365-370.

McAdams, C. J., and Maunsell, J. H. (1999). Effects of attention on the reliability of individual neurons in monkey visual cortex. Neuron 23, 765-773.

Naccache, L., Blandin, E., and Dehaene, S. (2002). Unconscious masked priming depends on temporal attention. Psychol. Sci. 13, 416-424.

Noesselt, T., Hillyard, S. A., Woldorff, M. G., Schoenfeld, A., Hagner, T., Jancke, L., Tempelmann, C., Hinrichs, H., and Heinze, H. J. (2002). Delayed striate cortical activation during spatial attention. Neuron 35, 575-587.

Osipova, D., Takashima, A., Oostenveld, R., Fernandez, G. Maris, E., and Jensen, O. (2006). Theta and gamma oscillations predict encoding and retrieval of declarative memory. J. Neurosci. 26, 7523-7531.

Pelli, D. G. (1997). The VideoToolbox software for visual psychophysics: transforming numbers into movies. Spat. Vis. 10, 437-442.

Pins, D., and Ffytche, D. (2003). The neural correlates of conscious vision. Cereb. Cortex 13, 461-474.

Poghosyan, V., and Ioannides, A. A. (2008). Attention modulates earliest responses in the primary auditory and visual cortices. Neuron 58 , 802-813.

Portin, K., Vanni, S., Virsu, V., and Hari, R. (1999). Stronger occipital cortical activation to lower than upper visual field stimuli. 
Neuromagnetic recordings. Exp. Brain Res. 124, 287-294.

Posner, M. I. (1994). Attention: the mechanisms of consciousness. Proc. Natl. Acad. Sci. U.S.A. 91, 7398-7403.

Posner, M. I., Snyder, C. R., and Davidson, B. J. (1980). Attention and the detection of signals. J. Exp. Psychol. 109, 160-174.

Reynolds, J. H., and Chelazzi, L. (2004). Attentional modulation of visual processing. Annu. Rev. Neurosci. 27, 611-647.

Reynolds, J. H., Pasternak, T., and Desimone, R. (2000). Attention increases sensitivity of V4 neurons. Neuron 26, 703-714.

Schurger, A., Cowey, A., Cohen, J. D., Treisman, A., and Tallon-Baudry, C. (2008). Distinct and independent correlates of attention and awareness in a hemianopic patient. Neuropsychologia 46, 2189-2197.

Sergent, C., Baillet, S., and Dehaene, S. (2005). Timing of the brain events underlying access to consciousness during the attentional blink. Nat. Neurosci. 8, 1391-1400.

Siegel, M., Donner, T. H., Oostenveld, R., Fries, P., and Engel, A. K. (2008). Neuronal synchronization along the dorsal visual pathway reflects the focus of spatial attention. Neuron 60, 709-719.

Simons, D. J., and Chabris, C. F. (1999). Gorillas in our midst: sustained inattentional blindness for dynamic events. Perception 28, 1059-1074.

Smith, P. L., and Ratcliff, R. (2009). An integrated theory of attention and decision making in visual signal detection. Psychol. Rev. 116, 283-317.

Smith, P. L., Ratcliff, R., and Wolfgang, B. J. (2004). Attention orienting and the time course of perceptual decisions: response time distributions with masked and unmasked displays. Vision Res. 44, 1297-1320.

Störmer, V. S., McDonald, J. J., and Hillyard, S. A. (2009). Cross-modal cueing of attention alters appearance and early cortical processing of visual stimuli. Proc. Natl. Acad. Sci. U.S.A. 106, 22456-22461.

Sumner, P., Tsai, P. C., Yu, K., and Nachev, P. (2006). Attentional modulation of sensorimotor processes in the absence of perceptual awareness. Proc. Natl. Acad. Sci. U.S.A. 103, 10520-10525.

Tallon-Baudry, C., Bertrand, O., Delpuech, C., and Permier, J. (1997). Oscillatory gamma-band $(30-70 \mathrm{~Hz})$ activity induced by a visual search task in humans. J. Neurosci. 17, 722-734.

Thorpe, S., Fize, D., and Marlot, C. (1996). Speed of processing in the human visual system. Nature 381, 520-522.

Thut, G., Nietzel, A., Brandt, S. A., and Pascual-Leone, A. (2006). Alphaband electroencephalographic activity over occipital cortex indexes visuospatial attention bias and predicts visual target detection. J. Neurosci. 26, 9494-9502.

Tyler, C. W. (1997). Colour bitstealing to enhance the luminance resolution of digital displays on a single pixel basis. Spat. Vis. 10, 369-377.

van Boxtel, J. J., Tsuchiya, N., and Koch, C. (2010). Opposing effects of attention and consciousness on afterimages. Proc. Natl. Acad. Sci. U.S.A. 107, 8883-8888.

van Dijk, H., Schoffelen, J. M. Oostenveld, R., and Jensen, O. (2008). Prestimulus oscillatory activity in the alpha band predicts visual discrimination ability. J. Neurosci. 28, 1816-1823.

VanRullen, R., and Thorpe, S. J. (2001). The time course of visual processing: from early perception to decision-making. J. Cogn. Neurosci. 13, 454-461.

VanRullen, R., and Thorpe, S. J. (2002). Surfing a spike wave down the ventral stream. Vision Res. 42, 2593-2615.

Vorberg, D., Mattler, U., Heinecke, A., Schmidt, T., and Schwarzbach, J. (2003). Different time courses for visual perception and action priming. Proc. Natl. Acad. Sci. U.S.A. 100 , 6275-6280.

Weiskrantz, L. (1986). Blindsight: A Case Study and Implications. Oxford: Oxford University Press.

Woodman, G. F., and Luck, S. J. (2003). Dissociations among attention, perception, and awareness during object-substitution masking. Psychol. Sci. 14, 605-611.

Worden, M. S., Foxe, J. J., Wang, N., and Simpson, G. V. (2000). Anticipatory biasing of visuospatial attention indexed by retinotopically specific alpha-band electroencephalography increases over occipital cortex. J. Neurosci. 20, RC63.

Wyart, V., and Tallon-Baudry, C. (2008). Neural dissociation between visual awareness and spatial attention. J. Neurosci. 28, 2667-2679.

Wyart, V., and Tallon-Baudry, C. (2009). How ongoing fluctuations in human visual cortex predict perceptual awareness: baseline shift versus decision bias. J. Neurosci. 29, 8715-8725.

Conflict of Interest Statement: The authors declare that the research was conducted in the absence of any commercial or financial relationships that could be construed as a potential conflict of interest.

Received: 15 November 2011; paper pending published: 16 December 2011; accepted: 25 January 2012; published online: 10 February 2012.

Citation: Wyart V, Dehaene $S$ and Tallon-Baudry C (2012) Early dissociation between neural signatures of endogenous spatial attention and perceptual awareness during visual masking. Front. Hum. Neurosci. 6:16. doi: 10.3389/fnhum.2012.00016

Copyright (c) 2012 Wyart, Dehaene and Tallon-Baudry. This is an open-access article distributed under the terms of the Creative Commons Attribution Non Commercial License, which permits noncommercial use, distribution, and reproduction in other forums, provided the original authors and source are credited. 